\title{
Prevalence of stroke and related burden among older people living in Latin America, India and China
}

Cleusa P Ferri, ${ }_{5}^{1}$ Claudia Schoenborn, ${ }^{1}$ Lalit Kalra, ${ }^{2}$ Daisy Acosta, ${ }^{3}$ Mariella Guerra, ${ }^{1,4}$ Yueqin Huang, ${ }^{5}$ K S Jacob, ${ }^{6}$ Juan J Llibre Rodriguez, ${ }^{1,7}$ Aquiles Salas, ${ }^{8}$ Ana Luisa Sosa, ${ }^{1,9}$ Joseph D Williams, ${ }^{10}$ Zhaorui Liu, ${ }^{5}$ Tais Moriyama, ${ }^{11}$ Adolfo Valhuerdi, ${ }^{12}$ Martin J Prince ${ }^{1}$

For numbered affiliations see end of article.

\section{Correspondence to}

Dr Cleusa P Ferri, Section of Epidemiology (Box 60), Institute of Psychiatry (King's College London), De Crespigny Park London SE5 8AF, UK cleusa.ferri@kcl.ac.uk

The first two authors should be regarded as joint first authors.

Received 5 November 2010 Revised 10 February 2011

Accepted 14 February 2011

Published Online First

14 March 2011

\section{ABSTRACT}

Objectives Despite the growing importance of stroke in developing countries, little is known of stroke burden in survivors. The authors investigated the prevalence of selfreported stroke, stroke-related disability, dependence and care-giver strain in Latin America (LA), China and India.

Methods Cross-sectional surveys were conducted on individuals aged $65+(n=15022)$ living in specified catchment areas. Self-reported stroke diagnosis, disability, care needs and care giver burden were assessed using a standardised protocol. For those reporting stroke, the correlates of disability, dependence and care-giver burden were estimated at each site using Poisson or linear regression, and combined meta-analytically.

Results The prevalence of self-reported stroke ranged between $6 \%$ and $9 \%$ across most LA sites and urban China, but was much lower in urban India (1.9\%), and in rural sites in India (1.1\%), China (1.6\%) and Peru (2.7\%). The proportion of stroke survivors needing care varied between $20 \%$ and $39 \%$ in LA sites but was higher in rural China (44\%), urban China (54\%) and rural India (73\%). Comorbid dementia and depression were the main correlates of disability and dependence.

Conclusion The prevalence of stroke in urban LA and Chinese sites is nearly as high as in industrialised countries. High levels of disability and dependence in the other mainly rural and less-developed sites suggest underascertainment of less severe cases as one likely explanation for the lower prevalence in those settings. As the health transition proceeds, a further increase in numbers of older stroke survivors is to be anticipated. In addition to prevention, stroke rehabilitation and long-term care needs should be addressed.

\section{INTRODUCTION}

After ischaemic heart disease, stroke is the commonest cause of mortality worldwide ${ }^{1}$ having caused around 5.7 million deaths in $2005,{ }^{2}{ }^{3}$ of which more than $80 \%$ occurred in low- and middleincome countries (LMIC). In addition, estimates showed that there may be 62 million survivors of stroke worldwide, ${ }^{3}$ many of whom are likely to be disabled, ${ }^{2}$ with up to a third living with severe disability. ${ }^{4}$ Stroke incidence has declined by over $40 \%$ in the past four decades in high-income countries (HIC), but over the same period, incidence has doubled in LMIC. ${ }^{5}$ Early stroke death is decreasing in both LMIC and HIC. ${ }^{5}$ Rapid demographic ageing in LMIC, coupled with increasing stroke incidence and decreasing case death, will increase the relative burden in LMIC. Stroke-related mortality is well described in LMIC, but little is known about burden among survivors. Most estimates of stroke-related disability, dependence and care needs have been extrapolated from HIC studies. Furthermore, the few studies based in LMIC are limited by variable methods used to ascertain cases, the different periods of data collection $^{6}$ and the paucity of data on older people. According to the Global Burden of Disease project, ${ }^{4}$ $83 \%$ of stroke-related deaths and $56 \%$ of strokerelated DALYs in LMIC occurred among people aged 60 years and over.

Our population-based survey ${ }^{7}$ was designed to make valid comparisons across countries and cultures, comprising data on chronic diseases from over 15000 participants in 11 catchment areas in five Latin American countries, India and China, using the same protocol, definitions and assessment tools. In this study, we focus on three main aspects of stroke epidemiology in LMIC: the prevalence of self-reported stroke; the nature of social patterning in terms of the effects of age, sex and education on prevalence; and the extent of stroke-related disability, dependence and care-giver perceived strain, and their correlates.

\section{METHODS}

\section{Participants, settings and recruitment}

One-phase surveys were conducted of all residents aged 65 years and over in 11 catchment areas: urban sites in Cuba, Dominican Republic and Venezuela and urban and rural sites in Peru, Mexico, China and India. The target sample size was 2000 in each country, other than Cuba (3000). Response rates exceeded $80 \%$ in all sites, excepting urban China $(74 \%)$ and urban India (72\%). Baseline surveys included a wide-ranging participant interview, a structured clinical interview, a physical examination and an informant interview. We describe in detail the components that are relevant to the present study. A more detailed account of the study design and procedures is presented elsewhere. ${ }^{7}$

\section{Measures \\ Sociodemographics}

Participant's age, sex, educational level and number of household assets (used as a continuous variable).

\section{Health conditions}

1. Self-reported stroke was ascertained with the question 'have you ever had a stroke that needed 
medical attention?' If the answer was 'yes,' they were asked 'what happened?' The answer was coded positive only if the participant or informant gave a clear history of sudden onset of unilateral paralysis and/or loss of speech and/or blindness lasting for at least $24 \mathrm{~h}$; thus, previous transient ischaemic attacks were excluded. If the history was supportive of stroke, they were asked 'who diagnosed this stroke?' (no one/ primary healthcare worker/specialist). Stroke was coded only if a clinician had made the diagnosis.

2. Physical impairments; self-reported paralysis, weakness or loss of a limb; eyesight problems; stomach or intestine problems; arthritis or rheumatism; heart problems; hearing difficulties or deafness; breathlessness; difficulty breathing or asthma; faint or blackouts; skin disorders such as pressure sores, leg ulcers or severe burns; persistent cough were rated as present if they interfered with activities 'a little' or 'a lot. 8 The number of reported impairments was used as a continuous variable.

3. ICD-10 depressive episode ${ }^{9}$ in the month preceding the interview, derived using a computerised algorithm applied to a structured clinical mental state interview (the Geriatric Mental State (GMS)). ${ }^{10}$

4. Dementia was ascertained through multidomain cognitive testing and clinical and informant interview. Those meeting either the cross-culturally validated 10/66 dementia diagnosis algorithm $^{11}$ or the Diagnostic and Statistical Manual of Mental Disorders IV dementia criterion ${ }^{12}$ were considered to have dementia.

5. Disability assessed with the 12-item WHO Disability Assessment Schedule (WHODAS 2.0), for which unidimensionality and measurement invariance have previously been demonstrated. ${ }^{13}$ The 90th centile of the distribution in each site identified those with severe disability.

6. Dependence was ascertained from interviewer administered open-ended questions to a key informant. ${ }^{14}$ Codings (participant requiring no care, care some of the time or care much of the time) were based upon the interviewer's judgement of needs for care independent of whether these were routinely met. We dichotomised the variable into needing care and not needing care.

\section{Care-giver perceived strain}

The Zarit Burden Interview ${ }^{15}$ comprises 22 items that assess the carer's appraisal of the impact their involvement has had on their lives. For each item, burden is quantified from 0 (no burden) to 4 (highest burden).

\section{Ethics}

The study was approved by the local ethical committees in each country and by King's College London Research Ethics Committee. Participants were recruited following informed signed consent. Those who were unable to consent were recruited on the basis of a relative's signed agreement. Illiterate persons were read the information sheet and consent form, and invited to express their consent verbally, which was witnessed.

\section{Statistical analysis}

We used the 10/66 Dementia Research Group data archive (release 2.0) and STATA 10.0. All estimates were adjusted for household clustering and accompanied by robust 95\% CIs. When calculating effect sizes, we carried out analyses separately for each site and then used fixed-effects meta-analyses to combine them, with Higgins' $\mathrm{I}^{2}$ to estimate the degree of heterogeneity with approximate 95\% CIs. Where statistically significant, we present estimates from random-effect models.

\section{Prevalence of stroke}

We calculated the crude prevalence of self-reported stroke by sex, age group and site. Direct standardisation (for age, sex and education) was used to compare stroke prevalence among sites, with the whole sample as standard population. To compare with that from the USA, ${ }^{16}$ we used indirect standardisation for age and sex, calculating standardised morbidity ratios (SMRs) for each site. We used Poisson regression working models to calculate mutually adjusted prevalence ratios (PRs) for the effects of age, sex and education on stroke prevalence.

\section{Impact of stroke}

Restricting the sample to participants with self-reported stroke $(n=904)$, we modelled the effects of age, sex, education, household assets, dementia, depression and number of physical impairments on disability and care needs, again providing mutually adjusted PRs from a Poisson working model. Restricting the sample to informants who were providing care $(n=298)$, we used linear regression to estimate the effects of age, gender, education, number of household assets, dementia, depression, number of physical impairments, disability, carer age and sex on carer strain (Zarit total score). Effects were expressed as regression coefficients with robust $95 \%$ CIs.

\section{RESULTS}

We interviewed 15022 participants aged over 65 years of age, of whom 904 (497 women and 406 men) reported stroke. Demographic ageing was more advanced in the Latin American sites and urban China, than in rural China and India (table 1). Women preponderated in all sites. Educational levels were highest in Cuba, urban Peru and Venezuela. In the Dominican Republic, rural Mexico, rural China and India, most participants had not completed primary education. In these same sites, pension coverage was low and food insecurity high.

\section{Prevalence of stroke}

The crude prevalence of stroke varied considerably between sites, and was generally higher in urban compared with rural areas (table 2). With the exception of rural Peru, the Latin American sites had a similar prevalence, ranging from $6.5 \%$ in rural Mexico to $8.4 \%$ in the Dominican Republic. Urban China had the highest prevalence of stroke (9.3\%). Much lower prevalences were found in rural India (1.1\%), urban India $(1.9 \%)$, rural China (1.6\%) and rural Peru (2.7\%). Direct standardisation for age, sex and education had little effect on the variation among sites. Age- and sex-standardised morbidity ratios showed that the prevalence in urban Latin American sites was generally at least three-quarters that in the USA. ${ }^{16}$ SMRs for rural Peru, India and rural China were strikingly low (around one-quarter the prevalence in the USA), while the prevalence in urban China $(S M R=106)$ was very similar to the USA reference population. In rural India and urban Mexico, all self-reported strokes had been diagnosed by a clinician. The proportion not diagnosed by a clinician was low in urban China $(0.9 \%)$, the Dominican Republic (3.4\%), Cuba (3.5\%) and urban India (5.0\%). A much higher proportion of reported strokes were undiagnosed by clinicians in rural China (11.1\%), Venezuela (11.8\%), rural Mexico (12.2\%), urban (17.1\%) and rural (25.0\%) Peru.

Table 3 shows the effects of age, sex and education on stroke prevalence. Meta-analysed estimates showed that males were 


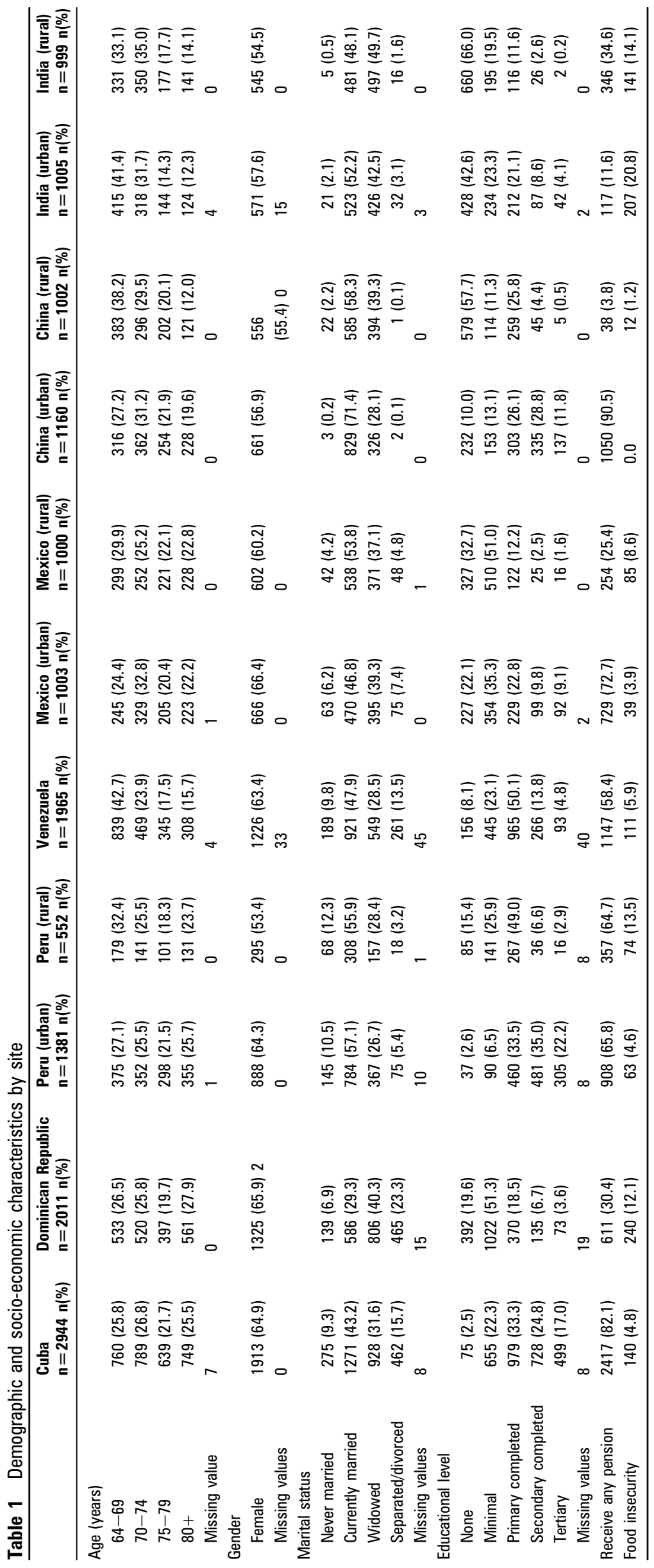


Table 2 Prevalence of self-reported stroke by age group, sex, and site; crude and standardised prevalence among those aged 65 and over, and standardised morbidity ratio

\begin{tabular}{|c|c|c|c|c|c|c|c|}
\hline & $\begin{array}{l}\text { Percentage } \\
65-69 \text { years } \\
(95 \% \mathrm{CI})\end{array}$ & $\begin{array}{l}\text { Percentage } \\
70-74 \text { years } \\
(95 \% \text { Cl })\end{array}$ & $\begin{array}{l}\text { Percentage } \\
75-79 \text { years } \\
(95 \% \mathrm{Cl})\end{array}$ & $\begin{array}{l}\text { Percentage } \\
80+\text { years } \\
(95 \% \mathrm{Cl})\end{array}$ & $\begin{array}{l}\text { Crude prevalence } \\
\text { percentage } \\
(95 \% \mathrm{CI})\end{array}$ & $\begin{array}{l}\text { Standardised } \\
\text { prevalence } \\
\text { percentage }(95 \% \mathrm{CI})\end{array}$ & $\begin{array}{l}\text { Standarized morbidity } \\
\text { ratio } † \text { percentage } \\
(95 \% \mathrm{Cl})\end{array}$ \\
\hline \multicolumn{8}{|c|}{ Cuba $(\mathrm{n}=2934 ; m v=9)$} \\
\hline Female & $4.5(2.7$ to 6.4$)$ & 6.6 (4.4 to 8.8$)$ & $6.6(4.2$ to 9.0$)$ & 9.0 (6.5 to 11.5$)$ & $7.6(6.6$ to 8.5$)$ & $7.3(6.0$ to 8.6$)$ & $84(73$ to 95$)$ \\
\hline Male & $5.9(3.1$ to 8.7$)$ & $9.2(5.9$ to 12.6$)$ & 7.9 (4.4 to 11.5$)$ & $14.2(9.6$ to 18.7$)$ & & & \\
\hline \multicolumn{8}{|c|}{ Dominican Republic $(\mathrm{n}=2002 ; \mathrm{mv}=6)$} \\
\hline Female & $4.4(2.2$ to 6.5$)$ & $8.7(5.6$ to 11.8$)$ & $5.3(2.6$ to 8.0$)$ & 10.7 (7.7 to 13.8$)$ & $8.4(7.2$ to 9.6$)$ & $8.0(6.7$ to 9.4$)$ & 93 (80 to 108$)$ \\
\hline Male & $7.0(3.3$ to 10.7$)$ & $13.8(8.9$ to 18.7$)$ & $7.6(3.0$ to 12.1$)$ & $11.9(6.9$ to 16.9$)$ & & & \\
\hline \multicolumn{8}{|c|}{ Peru (urban) $(n=1368 ; m v=8)$} \\
\hline Female & $3.8(1.5$ to 6.1$)$ & $8.2(4.5$ to 11.9$)$ & $4.2(1.3$ to 7.1$)$ & $5.2(2.2$ to 8.2$)$ & $6.8(5.4$ to 8.2$)$ & $6.5(4.1$ to 9.0$)$ & 75 (61 to 92$)$ \\
\hline Male & $7.2(2.3$ to 12.1$)$ & $8.4(3.6$ to 13.2$)$ & $8.6(3.2$ to 14.1$)$ & $12.9(7.3$ to 18.6$)$ & & & \\
\hline \multicolumn{8}{|c|}{ Peru (rural) $(\mathrm{n}=549 ; \mathrm{mv}=2)$} \\
\hline Female & $2.0(0.0$ to 4.8$)$ & $2.4(0.0$ to 5.8$)$ & 0.0 & $3.4(0.0$ to 8.1$)$ & $2.7(1.4$ to 4.1$)$ & $3.3(1.9$ to 4.8$)$ & $31(18$ to 51$)$ \\
\hline Male & $2.6(0.0$ to 6.1$)$ & $6.9(1.2$ to 13.6$)$ & $4.2(0.0$ to 10.0$)$ & 1.4 (0.0 to 4.2$)$ & & & \\
\hline \multicolumn{8}{|c|}{ Venezuela $(n=1905 ; m v=45)$} \\
\hline Female & $4.1(2.4$ to 5.9$)$ & $7.3(4.3$ to 10.3$)$ & 7.1 (3.6 to 10.6$)$ & $8.0(4.2$ to 11.8$)$ & $6.2(5.1$ to 7.3$)$ & $7.9(6.2$ to 9.5$)$ & 76 (63 to 91$)$ \\
\hline Male & $4.5(2.2$ to 6.8$)$ & $7.9(3.9$ to 12.0$)$ & $7.4(2.7$ to 12.1$)$ & $9.1(3.0$ to 15.2$)$ & & & \\
\hline \multicolumn{8}{|c|}{ Mexico (urban) $(n=1003 ; m v=0)$} \\
\hline Female & $3.8(1.0$ to 6.6$)$ & $8.1(4.3$ to 11.8$)$ & $4.0(0.5$ to 7.4$)$ & 5.6 (1.8 to 9.4$)$ & $6.7(5.1$ to 8.2$)$ & $6.5(4.9$ to 8.1$)$ & $76(60$ to 97$)$ \\
\hline Male & $5.0(0.0$ to 10.7$)$ & $6.8(2.2$ to 11.4$)$ & $8.9(2.4$ to 15.3$)$ & 15.0 (7.1 to 22.9$)$ & & & \\
\hline \multicolumn{8}{|c|}{ Mexico (rural) $(n=999 ; m v=0)$} \\
\hline Female & $6.6(3.1$ to 10.1$)$ & $9.4(4.7$ to 14.1$)$ & $6.7(2.4$ to 11.0$)$ & $3.3(0.1$ to 6.5$)$ & $6.5(5.0$ to 8.0$)$ & $6.8(4.7$ to 9.0$)$ & 73 (57 to 93 ) \\
\hline Male & $2.0(0.0$ to 4.7$)$ & $7.8(2.5$ to 13.1$)$ & $9.2(3.0$ to 15.4$)$ & 6.6 (1.8 to 11.4$)$ & & & \\
\hline \multicolumn{8}{|c|}{ China (urban) $(n=1160 ; m v=0)$} \\
\hline Female & 4.4 (1.6 to 7.2 ) & $8.9(5.0$ to 12.9$)$ & $7.3(2.9$ to 11.7$)$ & $7.6(2.7$ to 12.4$)$ & $9.3(7.6$ to 11.0$)$ & $9.1(7.3$ to 10.9$)$ & 106 (88 to 128) \\
\hline Male & 7.1 (2.3 to 12.0$)$ & $9.9(5.3$ to 14.6$)$ & 11.11 (5.3 to 16.9$)$ & $22.9(14.9$ to 30.9$)$ & & & \\
\hline \multicolumn{8}{|c|}{ China (rural) $(n=1002 ; m v=0)$} \\
\hline Female & $1.0(0.0$ to 2.5$)$ & $1.8(0.0$ to 3.9$)$ & $0.8(0.0$ to 2.4$)$ & 0.0 & $1.6(0.8$ to 2.4$)$ & $1.2(0.5$ to 2.0$)$ & $19(12$ to 31$)$ \\
\hline Male & $2.6(0.3$ to 4.9$)$ & $1.5(0.0$ to 3.6$)$ & $2.6(0.0$ to 6.2$)$ & $2.2(0.0$ to 6.5$)$ & & & \\
\hline \multicolumn{8}{|c|}{ India (urban) $(n=1004 ; m v=1)$} \\
\hline Female & $0.4(0.0$ to 1.2$)$ & $1.1(0.0$ to 2.5$)$ & $4.0(0.0$ to 8.5$)$ & $1.5(0.0$ to 4.5$)$ & $1.9(1.0$ to 2.7$)$ & $2.1(0.8$ to 3.5$)$ & 24 (15 to 38$)$ \\
\hline Male & $3.5(0.7$ to 6.2$)$ & $1.6(0.0$ to 3.8$)$ & $3.0(0.0$ to 7.2$)$ & $1.9(0.0$ to 5.8$)$ & & & \\
\hline \multicolumn{8}{|c|}{ India (rural) $(\mathrm{n}=999 ; \mathrm{mv}=0)$} \\
\hline Female & $2.6(0.3$ to 4.9$)$ & $0.5(0.0$ to 1.5$)$ & 0.0 & $1.5(0.0$ to 4.4$)$ & $1.1(0.4$ to 1.7$)$ & $0.6(0.2$ to 1.1$)$ & 13 (7 to 24$)$ \\
\hline Male & $1.4(0.0$ to 3.4$)$ & 0.0 & $1.1(0.0$ to 3.4$)$ & $1.4(0.0$ to 4.1$)$ & & & \\
\hline
\end{tabular}

*Standardised for age, sex and education.

†Indirect standardised for age and sex using the USA population as a reference.

mv, missing values.

$1.32(95 \%$ CI 1.14 to 1.49$)$ times more likely to report a stroke than females, with minimal heterogeneity between sites $\left(\mathrm{I}^{2}=22.8 \%\right.$; $95 \%$ CI 0 to 62$)$. There was a positive association with age in nearly all sites, with statistically significant associations in Cuba, Dominican Republic, Venezuela, urban Mexico and urban China; the pooled PR was 1.02 (95\% CI 1.01 to 1.03) per year of age, with no heterogeneity between sites $\left(\mathrm{I}^{2}=0 \%\right.$; $95 \%$ CI 0 to 60$)$. We found a non-statistically significant inverse association between levels of education and stroke prevalence in most sites, with a pooled estimate of 0.92 (95\% CI 0.86 to 0.98 ) and no heterogeneity $\left(\mathrm{I}^{2}=0 \%\right.$; $95 \% \mathrm{CI}$ 0 to 60$)$.

Table 3 Associations of age, sex and education with stroke prevalence (prevalence ratios with $95 \%$ Cls), by site and pooled across sites

\begin{tabular}{llll}
\hline Country & Age (per year) & Sex (male vs female) & Education (per level) \\
\hline Cuba & $1.03(1.01$ to 1.05$)$ & $1.43(1.11$ to 1.84$)$ & $0.91(0.80$ to 1.03$)$ \\
Dominican Republic & $1.03(1.01$ to 1.05$)$ & $1.36(1.01$ to 1.83$)$ & $0.98(0.84$ to 1.14$)$ \\
Peru (urban) & $1.01(0.82$ to 1.04$)$ & $1.73(1.18$ to 2.55$)$ & $1.02(0.82$ to 1.26$)$ \\
Peru (rural) & $0.99(0.93$ to 1.05$)$ & $1.82(0.67$ to 5.10$)$ & $0.90(0.43$ to 1.87$)$ \\
Venezuela & $1.02(1.00$ to 1.05$)$ & $1.14(0.79$ to 1.64$)$ & $0.83(0.66$ to 1.03$)$ \\
Mexico (urban) & $1.03(1.00$ to 1.07$)$ & $1.56(0.98$ to 2.47$)$ & $1.01(0.82$ to 1.24$)$ \\
Mexico (rural) & $1.00(0.97$ to 1.03$)$ & $0.94(0.58$ to 1.52$)$ & $1.07(0.78$ to 1.45$)$ \\
China (urban) & $1.04(1.01$ to 1.07$)$ & $1.95(1.35$ to 2.84$)$ & $0.87(0.76$ to 1.01$)$ \\
China (rural) & $0.95(0.86$ to 1.05$)$ & $2.70(0.96$ to 7.57$)$ & $0.68(0.42$ to 1.10$)$ \\
India (urban) & $1.02(0.95$ to 1.09$)$ & $2.01(0.78$ to 5.21$)$ & $1.06(0.74$ to 1.52$)$ \\
India (rural) & $0.96(0.83$ to 1.11$)$ & $0.59(0.20$ to 1.73$)$ & $1.21(0.64$ to 2.30$)$ \\
Pooled estimate & $1.02(1.01$ to 1.03$)$ & $1.32(1.14$ to 1.49$)$ & $0.92(0.86$ to 0.98$)$ \\
$\chi^{2}$ test for heterogeneity & $9.9(\mathrm{df}=10), \mathrm{p}=0.46$ & $13.0(\mathrm{df}=10), \mathrm{p}=0.23$ & $7.4(\mathrm{df}=10), \mathrm{p}=0.69$ \\
Higgins $\left.\right|^{2}$ percentage $(95 \% \mathrm{CI})$ & $0(0$ to 60$)$ & $23(0$ to 62$)$ & 0 (0 to 60$)$ \\
\hline
\end{tabular}




\section{Disability and care needs}

Among stroke survivors, between 18.5\% (rural Mexico) and $72.7 \%$ (rural India) had severe disability (table 4). Dementia, present in $25.4 \%$ of stroke survivors, was the strongest correlate of severe disability (pooled $\mathrm{PR}=2.63$; 95\% CI 1.89 to 3.37 ) (table 5). Depression was present in $10.8 \%$ and was also a strong correlate of disability (pooled $\mathrm{PR}=1.4095 \%$ CI 1.06 to 1.74). Disability increased with age (pooled $\mathrm{PR}=1.02$; 95\% CI 1.01 to 1.03 ) and number of physical impairments (pooled $\mathrm{PR}=1.12$; 95\% CI 1.07 to 1.16). Tests for heterogeneity were only statistically significant for dementia $\left(\chi^{2}=12.93 ; p=0.044, \mathrm{I}^{2}=54 \%\right.$; $95 \%$ CI 0 to 80 ). However, associations with dementia were positive and statistically significant in all sites, excepting rural India. The proportion of stroke survivors who reported inability or severe difficulty in standing for 30 min varied between sites (from $13.3 \%$ in rural Peru to $63.6 \%$ in rural India). The proportion reporting inability or severe difficulty in walking $1 \mathrm{~km}$ was higher (from 20.0\% in rural Peru to $63.6 \%$ in rural India). Incontinence was also highly prevalent, varying from $6.7 \%$ in rural Peru to $45.5 \%$ in rural India. The main correlates of dependence were similar to those for severe disability; other than that, depression was not associated.

We had information on needs for care for 882 of the 904 stroke survivors $(97.6 \%)$. In all, $305(34.6 \%)$ needed care. Of these, $74.4 \%$ needed care much of the time. The proportion needing care was similar in Latin American centres (from 20.0\% in rural Peru to $38.7 \%$ in urban Peru) but higher in urban China $(53.7 \%)$, rural China (43.7\%) and rural India (72.7\%) (table 4). Dementia was the strongest correlate of dependence (pooled $\mathrm{PR}=2.72,95 \%$ CI 2.21 to 3.24). The proportion needing care increased with age (pooled $\mathrm{PR}=1.03$; 95\% CI 1.01 to 1.04 ) and with the number of physical impairments ( $\mathrm{PR}=1.0895 \% \mathrm{CI} 1.03$ to 1.13). There was no heterogeneity between sites for these estimates, other than for number of assets ( $\mathrm{I}^{2}=58 \%$; $95 \%$ CI 3 to 82 ).

\section{Strain on carers of stroke survivors}

The proportion of carers $(n=286)$ who had to cut back on work to care for the person with stroke varied across sites (table 4). In Latin America, excepting rural Peru, the proportion varied from $20.0 \%$ in Venezuela to $57.1 \%$ in rural Mexico. The proportions were much higher in rural China $(71.4 \%)$ and rural India (87.5\%). In urban China, only $7.6 \%$ had cut back on work, but $20.0 \%$ had employed paid care givers. Increasing age was inversely associated with carer strain (table 5). Comorbid dementia made the largest contribution to carer strain, conferring a mean 7.1-point ( $95 \%$ CI 2.0 to 12.2 ) increase in the Zarit score. The effect of participant's disability on carer burden was a 0.15 increase on the Zarit scale for every one-point increase on the WHODAS 2.0 scale.

\section{DISCUSSION}

Over 15000 participants aged 65 years and over were interviewed in 11 sites in seven low- and middle-income countries. The prevalence of self-reported clinically diagnosed stroke was similar to that in the USA in urban China and in urban sites in Latin America, but significantly lower in rural Peru, rural China and India. Prevalence was higher among men, increased with age and decreased with education. Among the 904 stroke survivors, severe disability and dependence were common and were associated with comorbid dementia, depression and physical impairments. Carers often reported giving up work to care. Subjective carer strain was again associated with comorbid dementia but was lower for carers of older stroke survivors.

\section{Strengths of the study}

The main strength of this study is the use of a common protocol to study the prevalence of self-reported stroke, and other major chronic disease comorbidities across middle-income countries on three continents. Sampling focused exclusively on those aged 65 and over, among whom relatively little research had previously been conducted in these regions. The protocol paid particular attention to indicators of burden; disability, needs for care and strain among carers. Comparisons of stroke prevalence and burden are often hampered by methodological limitations, particularly study-design heterogeneity.

\section{Limitations}

\section{Generalisability}

Our studies were conducted in specific urban or rural catchment areas, and our findings may not therefore be safely generalised beyond their borders. While the communities may be considered to be typical in many ways of other similar urban or rural settings in the country concerned, they should not be considered to be representative of the country as a whole. Regional variation in patterns of morbidity may be considerable, relating for example to compositional differences in socio-economic status and underlying risk behaviours.

\section{Validity of the stroke ascertainment}

We ascertained stroke by self-report without confirmation by clinical examination, or access to medical records. Unfortunately, we did not have the opportunity to prevalidate this stroke ascertainment procedure in our study settings. Validation studies of self-report of stroke have shown variable results. One of the few studies to use cerebral CT to validate self-reported stroke estimated a sensitivity of $80 \%$ and a specificity of $99 \%$, with a PPV of $79 \% .{ }^{17}$ False-positive rates for screening procedures in prevalence studies range widely between $15 \%$ and $47 \%$; however, rates as high as $90 \%$ have been reported. ${ }^{18}$ Most false-positives arise from the failure to exclude TIAs. ${ }^{17}$ Several previous validation studies used self-completed screening questionnaires only as initial ascertainment, with no clinical interview to clarify the salience of reported symptoms. ${ }^{19} 20$ We hoped to have minimised this source of misclassification by training interviewers to ensure that stroke was only coded when the history was compatible with a diagnosis of stroke, with symptoms lasting at least $24 \mathrm{~h}$. Ideally, we would have tested formally the inter-rater reliability of this semistructured procedure between interviewers, and across sites, but again resource limitations precluded this possibility. We tried to increase specificity further by coding stroke only if it was reported that this had been diagnosed by a clinician. This stratagem might be expected to reduce sensitivity in resource-poor settings with low awareness and limited access to services. While relatively few participants reported stroke that had not been clinically diagnosed, particularly in least-resourced settings, this does not exclude the possibility of selective underreporting. People living in areas of better education, with better access to health services may be more likely to perceive their own morbidities, and to understand and recall clinician diagnoses, when compared with people in less advantaged settings, who may perceive symptoms as a normal part of life. ${ }^{21}$ This could partially explain the trends towards a positive association between level of education and prevalence of self-reported stroke observed in rural sites and in India, and for the very low overall prevalence observed in these sites.

\section{Sample size and precision}

Individual catchment area samples sizes were smaller in countries with a split urban/rural recruitment (ranging from 532 to 


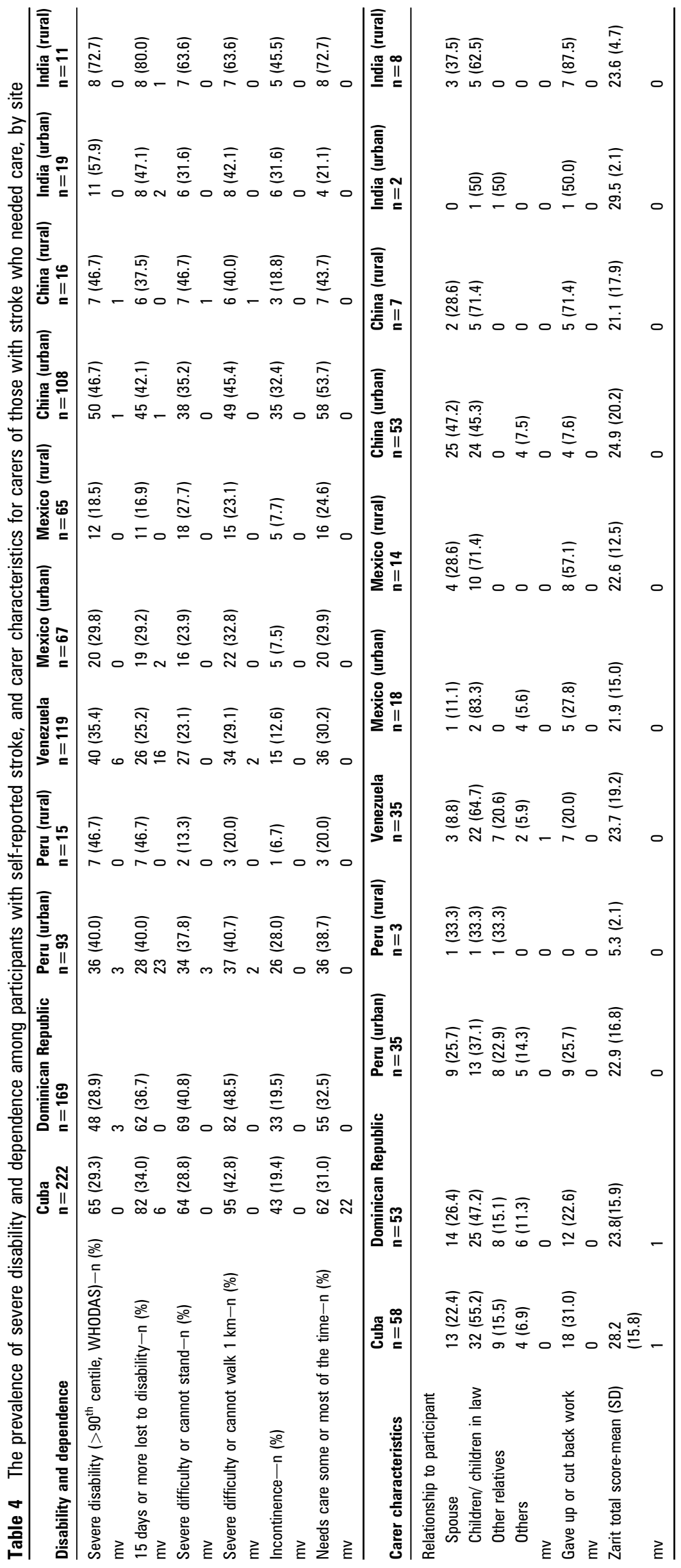




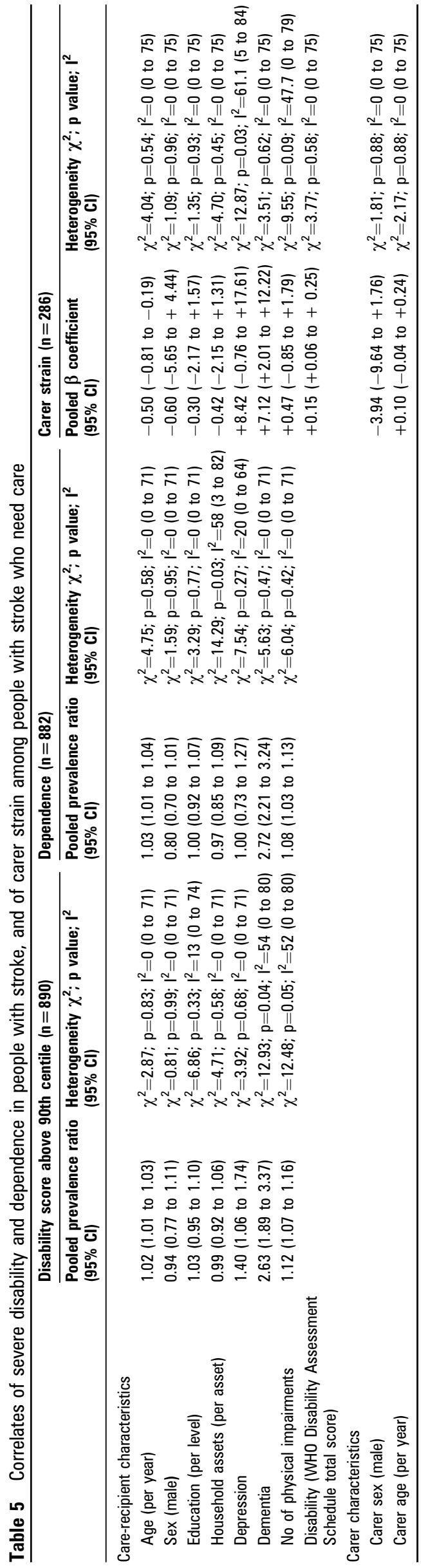

1381) than in those with a single urban catchment area (1965 to 2944). Our studies were not powered on the stroke outcome. The precision conferred is summarised in the width of the $95 \%$ CIs for the prevalence estimates, which is around 3\% ( $\pm 1.5 \%)$ for the smaller catchment areas, and $2 \%( \pm 1 \%)$ for the larger. Hence, imprecision was not a major problem, other than when considering characteristics of the smaller subset of participants reporting stroke (table 4).

\section{Contextualisation with previous research}

In general, estimates of stroke prevalence from Latin America have been lower than those from industrialised countries. ${ }^{22}$ In rural Bolivia in the early 1990s, ${ }^{6}$ the prevalence of stroke in people aged 65 years and over was $1.9 \%$, similar to our estimate from rural Peru $(2.7 \%)$ but much lower than that for rural Mexico (6.5\%). The prevalence among those aged 60 and over, between the early 1980s and 1990s, ranged from $2.5 \%$ in urban Chile, to $3.6 \%$ in Ecuador and $4.7 \%$ in Colombia. ${ }^{6}$ Direct comparison with our estimates is hampered by slightly different age groups of the study populations, and by the fact that our surveys were conducted 15-20 years later; nevertheless, we have found a higher prevalence of stroke among older people in Latin America than previously reported. A recent review of stroke epidemiology in China $^{23}$ found a decreasing north-south gradient of stroke incidence and prevalence. Only three studies

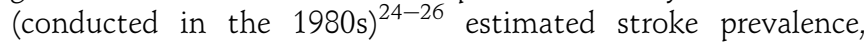
which was higher in urban compared with rural areas. This pattern, probably reflecting the progress of the health transition in China, is consistent with our finding of a much lower prevalence of stroke in rural compared with urban Beijing, a striking difference given that the two catchment area communities are only $100 \mathrm{~km}$ apart. In a study conducted in urban and rural Beijing in 1992 and repeated in 2000, there was an increase in total stroke prevalence among those aged 65 and over from $8.0 \%$ to $13.7 \%$, accompanied by a decrease in the urban/rural gradient. ${ }^{27}$ This latter finding was replicated in a survey of urban, suburban and rural areas in $2002 .{ }^{28}$ There are few studies on stroke prevalence from India. A study carried out in Kolkata between 2003 and $2005^{29}$ indicated a prevalence of stroke which is higher (3.4\% among those aged 60 and over) than we observed in Chennai (1.9\% among those aged 65 and over). The Kolkata study adopted a detailed screening assessment, with high sensitivity and specificity, and a second-phase neurologist assessment of screen positives and a random $10 \%$ of screen negatives with weighting back of final estimates. In two further Indian studies among Parsis in Mumbai, ${ }^{30}$ and in Kashmir, ${ }^{31}$ the prevalence was much lower. However, these studies were carried out in the mid-1980s and used weaker ascertainment procedures. In general, knowledge and awareness of stroke in India are very poor, ${ }^{32}$ which may account for a low prevalence in studies based on self-report of clinical diagnoses.

In a previous analysis of the 10/66 sample published in the Lancet in $2009,{ }^{33}$ stroke and limb impairments were found to be the second and third largest contributors to disability, after dementia. In the current study, we found that one-third of stroke survivors suffered from significant disability, and comorbid dementia and depression were important correlates. Dementia and depression by themselves are major contributors to disability worldwide in older adults ${ }^{4}$ and prevalent among people with stroke $(24.3 \%$ of stroke survivors were diagnosed as having dementia, and $10.8 \%$ had depression in our sample). In our study, severe disability among those with stroke also increased with number of comorbid physical impairments. Disability in stroke survivors may be the consequence of other 
chronic diseases, comorbidity being especially common in older people, but they may also be a consequence of worsening functional capacity arising from an interaction between the stroke and pre-existing physical, cognitive or psychological impairments. ${ }^{34}$ Our study was cross-sectional and cannot determine direction of causality. Prencipe et al found that the onset of stroke worsened functional capacity for nearly threequarters of those affected. ${ }^{34}$ We estimated that around one-third of stroke survivors needed care; similar proportions were observed in the Auckland study, ${ }^{35}$ although higher proportions have also been reported. ${ }^{36}$ In a large population-based study in Tanzania, all stroke survivors were cared for at home despite the fact that $21 \%$ of them were bedbound. ${ }^{37}$ Caring for stroke survivors can affect different aspects of an individual's wellbeing, including increased physical, mental and financial stress, particularly in more disadvantaged settings. ${ }^{38}$ In our study, around one-quarter of family carers for stroke survivors had had to stop or cut back on work to care. Little support is available from social and health services for long-term care and rehabilitation. It is therefore not surprising that the strain upon carers found in our study was high.

\section{CONCLUSION}

Prevalence of stroke among older people in our urban Latin American and Chinese sites, where risk factors such as unhealthy diet, sedentary lifestyle, smoking and diabetes have been increasing over the past few decades, was already nearly as high as in industrialised countries. Any bias in our estimates is likely to tend towards an underestimation of true prevalence, particularly in rural and less-developed sites. It is noteworthy that levels of disability and dependence among stroke survivors were particularly high in rural settings, suggesting underascertainment of less severe cases, rather than a high case death as an explanation for the large differences in observed prevalence. Alternatively, the incidence of stroke may still be relatively low in these settings, in which case, as the health transition proceeds, increases in numbers of older stroke survivors are to be anticipated. Stroke should be accorded a greater priority on the health agenda of these countries. Most stroke care in LMICs is symptomatic and provided by non-specialised teams. Patients are unlikely to be treated urgently; there is a lack of effective drugs, unsystematic use of available drugs and use of unproven medication. Rehabilitation is deficient due to lack of equipment and organisation of services. ${ }^{39}$ Home and traditional treatment of stroke is still accepted practice in rural areas of some LMICs. ${ }^{40}$ The example of HICs has shown that effective preventive strategies together with comprehensive acute stroke management and rehabilitation services can prevent millions of deaths from stroke, and improve the quality of life of those affected. ${ }^{41}$ It is important to target risk factors in LMICs, but this should be coupled with efforts to improve access to, and quality of, medical, rehabilitation and social care services for those already affected. The increasing burden of stroke is expected to be felt most keenly in LMICs; nonetheless, epidemiological research from these settings is scarce and outdated. More well-designed epidemiological studies are needed on stroke incidence (a focus for the incidence phase of the 10/66 studies ${ }^{7}$ ), prevalence, death, associated disability and long-term care needs, in order to plan for efficient and sustainable healthcare and prevention strategies.

\section{Author affiliations:}

${ }^{1}$ King's College London (Institute of Psychiatry), Health Service and Population Research Department, Section of Epidemiology, London, UK
${ }^{2}$ King's College London (Institute of Psychiatry), Clinical Neurosciences Division, London, UK

${ }^{3}$ Internal Medicine, Geriatric Section, Universidad Nacional Pedro Henriquez Ureña,

Santo Domingo, Dominican Republic

${ }^{4}$ Universidad Peruana Cayetano Heredia, Lima, Peru

${ }^{5}$ Division of Social Psychiatry and Behavioral Medicine, Peking University, Institute of Mental Health, Beijing, PR China

${ }^{6}$ Christian Medical College, Vellore, India

${ }^{7}$ Clinical Science Department, Medical University of Havana, Havana, Cuba

${ }^{8}$ Department of Medicine, Caracas University Hospital, Caracas, Venezuela

${ }^{9}$ National Institute of Neurology and Neurosurgery of Mexico, Mexico City, Mexico

${ }^{10}$ Community Health Department, VHS (Voluntary Health Services), Chennai, India

${ }^{11}$ Federal University of São Paulo, Department of Psychiatry, São Paulo, Brazil

${ }^{12}$ Internal Medicine Department, Universidad de Ciencias Medicas de Matanzas,

Matanzas, Cuba

Funding The 10/66 Dementia Research Group's research has been funded by the Wellcome Trust Health Consequences of Population Change Programme

(GR066133-Prevalence phase in Cuba and Brazil; GR08002-Incidence phase in

Peru, Mexico, Argentina, Cuba, Dominican Republic, Venezuela and China), the World Health Organization (India, Dominican Republic and China), the US Alzheimer's Association (IIRG-04-1286-Peru, Mexico and Argentina) and FONACIT/ CDCH/ UCV (Venezuela). The Rockefeller Foundation supported our dissemination meeting at their Bellagio Centre. Alzheimer's Disease International has provided support for networking and infrastructure.

Competing interests The 10/66 Dementia Research Group (DRG) works closely with Alzheimer's Disease International (ADI), the non-profit federation of 77 Alzheimer associations around the world. ADI is committed to strengthening Alzheimer associations worldwide, raising awareness regarding dementia and Alzheimer's disease, and advocating for more and better services for people with dementia and their care givers. ADI is supported in part by grants from GlaxoSmithKline, Novartis, Lundbeck, Pfizer and Eisai.

Ethics approval Ethics approval was provided by the local ethical committees in each country and by King's College London Research Ethics Committee.

Contributors All of the authors worked collectively to develop the protocols and methods described in this paper. MJP leads the 10/66 Dementia Research Group, and CPF acts as study coordinator. JJLR (Cuba), DA (Dominican Republic), MG (Peru), AS (Venezuela), ALS (Mexico), KSJ (Vellore, India), JDW (Chennai, India) and YH (China) are principal investigators responsible for the fieldwork in their countries. CPF and CS wrote the first draft of this manuscript and carried out the analyses. This was revised by MJP. All other authors reviewed the report, provided further contributions and suggestions, and approved the version submitted.

Provenance and peer review Not commissioned; externally peer reviewed.

\section{REFERENCES}

1. WHO. The World Health Report 2000. Health Systems: Improving Performance. Geneva: World Health Organization, 2000.

2. Adamson J, Beswick A, Ebrahim S. Is stroke the most common cause of disability? J Stroke Cerebrovasc Dis 2004;13:171-7.

3. Strong K, Mathers C, Bonita R. Preventing stroke: saving lives around the world. Lancet Neurol 2007;6:182-7.

4. WHO: World Health Organization. Global Burden of Disease-Deaths, Years of Life Lost and Years Lost Due to Disability. http://appswhoint/ghodata/?vid=72063 (acessed 26 Aug 2010).

5. Feigin VL, Lawes CM, Bennett DA, et al. Worldwide stroke incidence and early case fatality reported in 56 population-based studies: a systematic review. Lancet Neurol 2009;8:355-69.

6. Lavados PM, Hennis AJ, Fernandes JG, et al. Stroke epidemiology, prevention, and management strategies at a regional level: Latin America and the Caribbean. Lancet Neurol 2007;6:362-72.

7. Prince M, Ferri CP, Acosta D, et al. The protocols for the 10/66 dementia research group population-based research programme. BMC Public Health 2007;7:165.

8. George LK, Fillenbaum GG. OARS methodology. A decade of experience in geriatric assessment. J Am Geriatr Soc 1985;33:607-15.

9. World Health Organization. The ICD-10 Classification of Mental and Behavioral Disorders. Diagnostic Criterea for Research. WHO: Geneva, Switzerland, 1993.

10. Copeland JR, Prince M, Wilson KC, et al. The Geriatric Mental State Examination in the 21st century. Int J Geriatr Psychiatry 2002;17:729-32.

11. Prince $\mathbf{M}$, Acosta $\mathrm{D}$, Chiu $\mathrm{H}$, et al. Dementia diagnosis in developing countries: a cross-cultural validation study. Lancet 2003;361:909-17.

12. Prince MJ, de Rodriguez JL, Noriega L, et al. The 10/66 Dementia Research Group's fully operationalised DSM-IV dementia computerized diagnostic algorithm, compared with the 10/66 dementia algorithm and a clinician diagnosis: a population validation study. BMC Public Health 2008:8:219.

13. Sousa RM, Dewey ME, Acosta D, et al. Measuring disability across cultures-the psychometric properties of the WHODAS-II in older people from seven low- and 
midle-income countries. The 10/66 Dementia Research Group population-based survey. Int J Methods Psychiatr Res 2010;10:1-17.

14. Sousa RM, Ferri CP, Acosta D, et al. The contribution of chronic diseases to the prevalence of dependence among older people in Latin America, China and India: a 10/66 Dementia Research Group population-based survey. BMC Geriatr 2010;10:53.

15. Zarit SH, Todd PA, Zarit JM. Subjective burden of husbands and wives as caregivers: a longitudinal study. Gerontologist 1986;26:260-6.

16. National Heart, Lung, and Blood Institute (NHLBI). Morbidity and Mortality: 2009 Chart Book on Cardiovascular, Lung, and Blood Diseases, National Institutes of Health: USA. 2009.

17. Engstad T, Bonaa KH, Viitanen M. Validity of self-reported stroke: The Tromso Study. Stroke 2000;31:1602-7.

18. Di Carlo A, Candelise L, Gandolfo C, et al. Influence of different screening procedures on the stroke prevalence estimates: the Italian Longitudinal Study on Aging. Cerebrovasc Dis 1999;9:231-7.

19. Geddes JM, Fear J, Tennant A, et al. Prevalence of self reported stroke in a population in northern England. J Epidemiol Community Health 1996;50:140-3.

20. O'Mahony PG, Thomson RG, Dobson R, et al. The prevalence of stroke and associated disability. J Public Health Med 1999;21:166-71.

21. Sen A. Health: perception versus observation. BMJ 2002;324:860-1.

22. Saposnik G, Del Brutto OH. Stroke in South America: a systematic review of incidence, prevalence, and stroke subtypes. Stroke 2003;34:2103-7.

23. Liu M, Wu B, Wang WZ, et al. Stroke in China: epidemiology, prevention, and management strategies. Lancet Neurol 2007;6:456-64.

24. Li SC, Schoenberg BS, Wang CC, et al. Cerebrovascular disease in the People's Republic of China: epidemiologic and clinical features. Neurology 1985;35:1708-13.

25. Li ZS, Yang OD, Chen SM, et al. Epidemiological survey of cerebrovascular disease in rural areas of China. Zhong-Hua Shen-Jing Wai-ke Za-Zhi 1989;5:7-11.

26. Xue GB, Yu BX, Wang XZ, et al. Stroke in urban and rural areas of China. Chin Med J (Engl) 1991:104:697-704.

27. Fang $\mathbf{X H}$, Tang Zhe, Xiang MJ, et al. Trends in prevalence of stroke and strokerelated disability from 1992 to 2000 in Beijing people older than 55 years. Chin $J$ Geriatr Heart Brain Vessel Dis 2007:9.
28. Liu HJ, Fang XH, Zhang GF, et al. A comprehansive evaluation on stroke prevalence among elderly in urban and rural areas of Beijing, 2002. Zhonghua Liu Xing Bing Xue Za Zhi 2005:26:767-71.

29. Das SK, Biswas A, Roy J, et al. Prevalence of major neurological disorders among geriatric population in the metropolitan city of Kolkata. J Assoc Physicians India 2008:56:175-81.

30. Bharucha NE, Bharucha EP, Bharucha $A E$, et al. Prevalence of stroke in the Parsi community of Bombay. Stroke 1988;19:60-2.

31. Razdan S, Koul RL, Motta A, et al. Cerebrovascular disease in rural Kashmir, India. Stroke 1989;20:1691-3.

32. Pandian JD, Srikanth V, Read SJ, et al. Poverty and stroke in India: a time to act. Stroke 2007:38:3063-9.

33. Sousa RM, Ferri CP, Acosta D, et al. Contribution of chronic diseases to disability in elderly people in countries with low and middle incomes: a 10/66 Dementia Research Group population-based survey. Lancet 2009;374:1821-30.

34. Prencipe M, Ferretti C, Casini AR, et al. Stroke, disability, and dementia: results of a population survey. Stroke 1997;28:531-6.

35. Bonita R, Solomon N, Broad JB. Prevalence of stroke and stroke-related disability. Estimates from the Auckland stroke studies. Stroke 1997;28:1898-902.

36. Clark ID, Opit LJ. The prevalence of stroke in those at home and the need for care. J Public Health Med 1994;16:93-6.

37. Walker RW, McLarty DG, Masuki G, et al. Age specific prevalence of impairment and disability relating to hemiplegic stroke in the Hai District of northern Tanzania. Adult Morbidity and Mortality Project. J Neurol Neurosurg Psychiatry 2000:68:744-9.

38. Das S, Hazra A, Ray BK, et al. Burden among stroke caregivers: results of a community-based study from Kolkata, India. Stroke 2010;41:2965-8.

39. World Health Organisation. Neurological Disorders: Public Health Challenges. Genevā, 2006.

40. Poungvarin N. Stroke in the developing world. Lancet 1998;352(Suppl 3): SIII19-22.

41. Lopez AD, Mathers CD, Ezzati M, et al. Global and regional burden of disease and risk factors, 2001: systematic analysis of population health data. Lancet 2006:367:1747-57. 